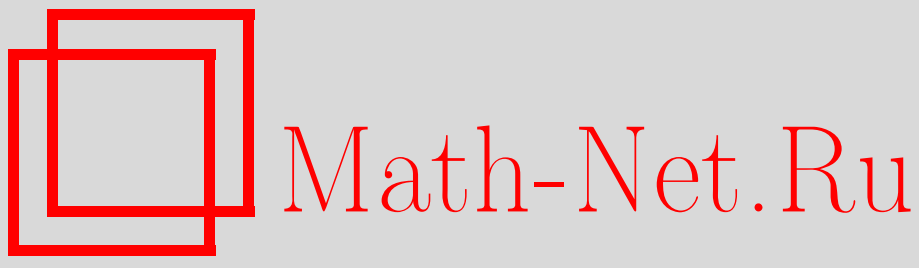

Г. В. Павлов, В. С. Бородин, Нутационные автопараметрические колебания вертикального ротора при обкатывании статора, Вестн. Сам. гос. техн. ун-та. Сер. Физ.-мат. науки, 2004, выпуск 26, 48-51

DOI: https://doi.org/10.14498/vsgtu176

Использование Общероссийского математического портала Math-Net.Ru подразумевает, что вы прочитали и согласны с пользовательским соглашением

http://www . mathnet.ru/rus/agreement

Параметры загрузки:

IP : 34.229 .45 .116

26 апреля 2023 г., 10:06:50

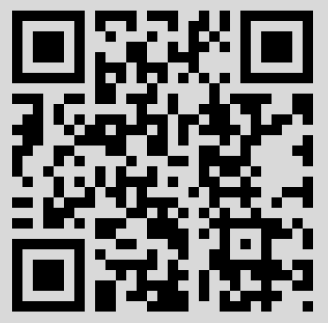




\section{НУТАЦИОННЫЕ АВТОПАРАМЕТРИЧЕСКИЕ КОЛЕБАНИЯ ВЕРТИКАЛЬНОГО РОТОРА ПРИ ОБКАТЫВАНИИ СТАТОРА}

Исследована задача о качении статически несбалансированного двухопорного вертикального ротора в режиме обкатывания внутренней поверхности статора. Найдены границь устойчивости режима и проведен численньй анализ уравнений движения ротора. Построень амплитудно-частотные и фазово-частотные характеристики.

Рассматривается качение статически несбалансированного двухопорного вертикального ротора, моделируемого тонким неоднородным диском на жестком валу, подвешенного в опорах системой горизонтальных пружин с жесткостями $C_{1 \alpha}, C_{1 \beta}, C_{2 \alpha}, C_{2 \beta}$, создающих упругое поле, вращающееся вместе с ротором. Вертикальное смещение ротора стеснено вертикальной пружиной с жесткостью $C_{z}$. Влияние внешнего трения учтено введением в опоры диссипативных сил с неполной диссипацией. Считаем, что при равновесии ротора диск касается цилиндра и, вообще говоря, отклоняется от оси цилиндра. Движение ротора происходит вне связи с двигателем, по инерции. Такой характер движения ротора может соответствовать аварийному режиму.

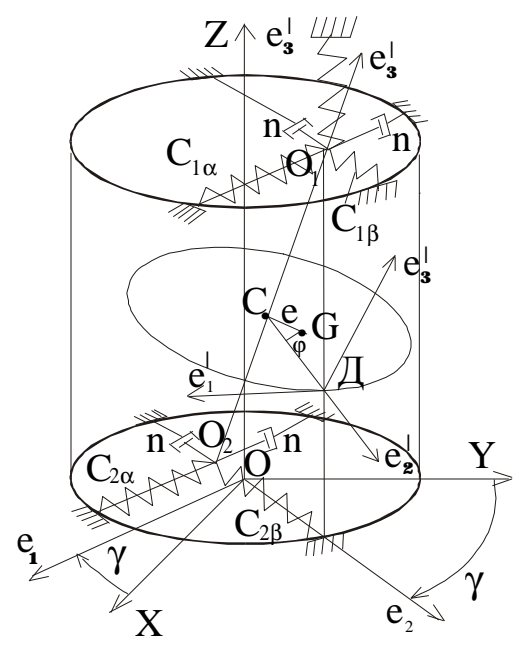

Р и с. 1. Схема качения ротора

Движение ротора отнесено к неподвижной системе координат OXYZ (рис. 1). Вертикальная ось $z$ совмещена с осью $e_{3}$, вращающегося репера $e_{1} e_{2} e_{3}$, ось $e_{2}$ которого лежит в одной вертикальной плоскости с точкой Д. Эта точка выбрана за начало полуподвижной системы $e_{1}^{\prime} e_{2}^{\prime} e_{3}^{\prime}$. Ось $e_{2}^{\prime}$ проходит через геометрический центр диска и точку Д, а $e_{1}^{\prime}-$ по касательной к диску в точке Д и направлена в сторону возрастания угла собственного вращения $\varphi$ диска. С центром масс диска совмещено начало жестко связанных с диском осей хуz. За обобщенные координаты приняты $\gamma, z$, следящие за точкой касания, и углы Резаля $\alpha, \beta$ и $\varphi$.

Задача в такой постановке приводит к трем динамическим нелинейным уравнениям движения и двум линейным по скоростям уравнениям неголономных связей [1]. Важным представляется анализ режима движения ротора, когда траектория точки касания лежит в плоскости параллельной основанию. В этом случае уравнения связей становятся интегрируемыми. Удерживая в уравнениях первые слагаемые разложения функций в ряды Тейлора относительно нулевых значений переменных, положим, для полноты исследования, что зависимость силы нелинейной упругой связи от перемещения в плоскости угла $\beta$ представлена функцией $f(\beta)=\gamma_{3} \beta^{3}+\gamma_{5} \beta^{5}$. Тогда система уравнений, описывающих движение ротора в окрестности локального минимума потенциальной энергии, определенной нулевыми значениями обобщенных координат принимает вид

$$
\begin{gathered}
\ddot{\beta}+\omega^{2}(\dot{\varphi}) \beta=-n_{1} z+\varepsilon f_{1}(\beta, \dot{\beta}, \ddot{\beta}, \dot{\varphi}, \dot{\varphi})+\varepsilon^{2} \ldots, \ddot{\varphi}=0, \dot{z}=0 . \\
\text { Здесь } \omega(\dot{\varphi})=\sqrt{\left[0.8\left(\frac{C_{22}}{M r^{2}}-\frac{r}{R} \dot{\varphi}^{2}\right)\right]} ; M-\text { масса диска ротора; } r-\text { радиус диска; } R-\text { радиус }
\end{gathered}
$$

цилиндра-статора; $\varepsilon=\frac{e}{r}, e-$ эксцентриситет диска; $n_{l}=0.8 \frac{C_{23}}{M r^{2}}$;

$$
f_{1}(\beta, \dot{\beta}, \ddot{\beta}, \dot{\varphi}, \dot{\varphi})=1.6\left(\ddot{\beta} \cos \varphi-\dot{\varphi} \dot{\beta} \sin \varphi-\frac{r}{2 R} \dot{\varphi}^{2} \beta \cos \varphi-k_{1} \dot{\beta}-\gamma_{3} \beta^{3}-\gamma_{5} \beta^{5}\right) ; k_{1}=0.8 \frac{n}{M r^{2}} ;
$$


$n$ - коэффициент внешнего трения; $C_{22}=C_{1 \beta} L_{1}^{2}+C_{2 \beta} L_{2}^{2}, C_{23}=C_{z} r, L_{1}, L_{2}-$ расстояния от опор до точки крепления диска. Как следует из (1) движение ротора сопровождается смешанными вынужденно автопараметрическими колебаниями, обусловленными статической неуравновешенностью и наличием слагаемого $\frac{r}{2 R} \dot{\varphi}^{2} \beta \cos \varphi$, вносящего параметрическое возмущение.

Характерна зависимость собственной частоты системы ротор упруго демпфирующие опоры от частоты вращения ротора, представленная на рис. 2. Первое уравнение (1) можно рассматривать как математическую модель маятника с вибрирующей верхней точкой подвеса, а при $\omega<0$, как модель колебания обращенного маятника в поле центробежных сил инерции. Интегралом третьего уравнения является константа. Полагаем ее равной нулю. Наличие ненулевого члена с постоянным значением координаты $z$ не повлияет на характер изменения амплитуды и фазы колебаний. Это приводит лишь к уводу центра колебаний, вследствие несимметричности функции $\beta(a, \Psi)$. Такое заключение нельзя считать очевидным, если $\dot{\varphi}=\Omega \neq$ const. Предполагая, что частота $\dot{\varphi}$

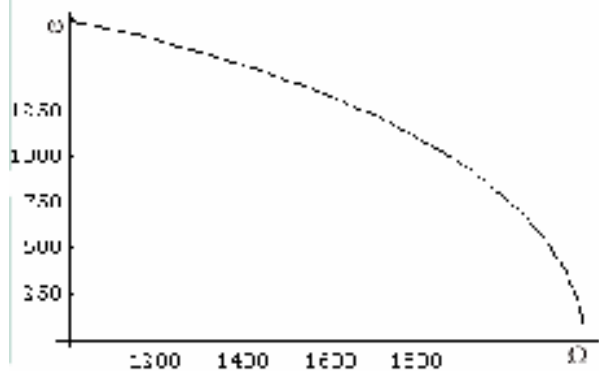

Р и с. 2. Зависимость собственной частоты системы от частоты вращения медленно изменяется в течение периода и что расстройка частот $\omega-p$ охватывает как резонансную зону, так и зоны подходов к резонансу, будем искать решение системы (1) в виде

$$
\beta=a \cos \Psi, \dot{\beta}=-a p \sin \Psi, \dot{\varphi}=\Omega,(\Psi=p t+\xi) .
$$

Здесь $\Omega$ - порождающее решение второго уравнения соответствующего системе (1). Неизвестная частота $p$ в выражении полной фазы $\Psi$ определяется, исходя из анализа фаз комбинационных частот функции $f_{l}(\beta, \dot{\beta}, \ddot{\beta}, \varphi, \dot{\varphi})$, стоящей под знаком интегралов [2]:

$$
\begin{aligned}
& \int_{0}^{2 \pi}\left[\frac{r}{R} \Omega^{2} \cos \Psi \cos \frac{\Omega}{p}(\Psi-\xi)-2 \omega \Omega \sin \Psi \sin \frac{\Omega}{p}(\Psi-\xi)\right] \sin \Psi d \Psi ; \\
& \int_{0}^{2 \pi}\left[\frac{r}{R} \Omega^{2} \cos \Psi \cos \frac{\Omega}{p}(\Psi-\xi)-2 \omega \Omega \sin \Psi \sin \frac{\Omega}{p}(\Psi-\xi)\right] \cos \Psi d \Psi .
\end{aligned}
$$

После преобразования произведения косинусов и синусов получаем подынтегральные выражения, содержащие тригонометрические функции с фазами (2 $\left.\frac{\mathrm{m}}{p}\right) \Psi \frac{\Omega}{p} \xi$. Отсюда следует, что при отношении $\frac{\Omega}{p}$ равному целому числу, для обнаружения синхронного резонанса в первом приближении, необходимо выполнение равенства $\Omega=2 p$. Следуя методу возмущений, амплитуду $a$, фазы $\xi$ и угловую скорость $\Omega$ определим из системы уравнений

$$
\frac{d a}{d t}=\varepsilon A_{l}(a, \xi, \Omega), \frac{d \xi}{d t}=\omega-p+\varepsilon B_{l}(a, \xi, \Omega), \frac{d \Omega}{d t}=\varepsilon C_{l}(a, \Omega),
$$

где функции $A_{1}, B_{1}$ находятся как частные, периодические по $\xi$ решения уравнений

$$
\begin{aligned}
& (\omega-p) a \frac{\partial B_{1}}{\partial \xi}+2 \omega A_{1}=-\frac{1}{\pi} \int_{0}^{2 \pi}\left[F_{1}(a \cos \Psi,-a p \sin \Psi, \Omega)+F_{2}\left(a \cos \Psi, \frac{\Omega}{p}(\Psi-\xi)\right)\right] \sin \Psi d \Psi ; \\
& (\omega-p) a \frac{\partial A_{1}}{\partial \xi}-2 a \omega B_{1}=\frac{1}{\pi} \int_{0}^{2 \pi}\left[F_{1}(a \cos \Psi,-a p \sin \Psi, \Omega)+F_{2}\left(a \cos \Psi, \frac{\Omega}{p}(\Psi-\xi)\right)\right] \cos \Psi d \Psi ; \\
& C_{1}=\frac{\Omega^{2}}{2 \pi} \int_{0}^{2 \pi} \sin \frac{\Omega}{p}(\Psi-\xi) d \Psi . \text { Здесь } F_{1}(a \cos \Psi,-a p \sin \Psi, \Omega)=-k_{1} \dot{\beta}-\gamma_{3} \beta^{3}-\gamma_{5} \beta^{5} ; \\
& F_{2}\left(a \cos \Psi, \frac{\Omega}{p}(\Psi-\xi)\right)=\ddot{\beta} \cos \varphi-\dot{\varphi} \dot{\beta} \sin \varphi-\frac{r}{2 R} \dot{\varphi}^{2} \beta \cos \varphi .
\end{aligned}
$$


Проводя усреднение при $\gamma_{5}=0$ по быстрой фазе $\psi$, запишем амплитудно-фазовые уравнения в явном виде:

$$
\frac{d a}{d t}=-\frac{n \Omega a}{5 M r^{2} \omega}-\frac{\Omega a}{10}\left(1-\frac{2 r}{R}\right) \sin 2 \xi ; \frac{d \xi}{d t}=\omega-\frac{\Omega}{2}+\frac{3 \gamma a^{2}}{10 \omega}-\frac{\Omega}{10}\left(1-\frac{2 r}{R}\right) \cos 2 \xi ; \frac{d \Omega}{d t}=0 .
$$

Слагаемое с гармонической составляющей вызывает субгармонические колебания, источником которых является, кроме нестационарной силы $\frac{r}{R} \dot{\varphi} \rho \cos \varphi$, диссипативная сила $2 \dot{\varphi} \dot{\beta} \sin \varphi$ и нестационарная сила инерции $\ddot{\beta} \cos \varphi$.

Первые слагаемые в правых частях (3) моделируют автоколебательный процесс. Анализ этой системы проведен при следующих численных данных, заимствованных из [3]

$$
C_{1 \alpha}=C_{1 \beta}=C_{2 \alpha}=C_{2 \beta}=3260 \kappa 2 c \bullet c^{2} * c^{-1}, \quad L_{1}=21 \mathrm{cM}, L_{2}=18.9 \mathrm{cM}, \mathrm{C}_{\mathrm{z}}=10000 \kappa 2 c \bullet c^{2} \bullet \mathrm{cm}^{-1} .
$$
$R=9.99 \mathrm{cM}, R=10 \mathrm{cM}, M=7.55 \cdot 10^{-3} \kappa 2 c \cdot c^{2} \cdot \mathrm{cM}^{-1}, n=0.5 \kappa 2 c \bullet c M \bullet c, \gamma_{3}=0.2 \kappa 2 c \bullet \mathrm{cM}^{-3}$.

В соответствии с (3) найдем уравнения стационарных амплитуд и фаз субгармонических колебаний центра масс ротора:

$$
\begin{aligned}
& a^{4}+\frac{20}{3} \frac{\omega}{\gamma}\left(\omega-\frac{\Omega}{2}\right) a^{2}+\frac{4}{9} \frac{n^{2} \Omega^{2}}{M^{2} r^{4} \gamma^{2}}+\frac{100}{9} \frac{\omega^{2}}{\gamma^{2}}\left(\omega-\frac{\Omega}{2}\right)^{2}-\frac{\omega^{2} \Omega^{2}}{10 \gamma^{2}}\left(1-\frac{2 r}{R}\right)^{2}=0 ; \\
& \operatorname{tg} 2 \xi=-\frac{n \Omega}{5 M r^{2} \omega\left(\omega-\frac{\Omega}{2}+\frac{3}{10} \frac{\gamma}{\omega} a^{2}\right)} ; \sin 2 \xi=-\frac{2 n R}{M r^{2} \omega(R-2 r)} .
\end{aligned}
$$

С точностью до $\varepsilon \approx \omega-\frac{\Omega}{2}$ имеем

$$
a^{2}=\frac{10}{3 \gamma}\left[\frac{\Omega^{2}}{4}-\omega^{2} \pm \frac{0.2}{M r^{2}} \sqrt{M^{2} r^{4} \omega^{4}\left(1-\frac{2 r}{R}\right)^{2}-4 n^{2} \omega^{2}}\right], \operatorname{tg} 2 \xi=-\frac{2 n \Omega}{3 M r^{2} \gamma a^{2}}
$$

Граница зоны синхронизации находится в интервале

$$
\omega^{2}-\frac{0.2}{M r^{2}} \sqrt{M^{2} r^{4} \omega^{4}\left(1-\frac{2 r}{R}\right)^{2}-4 n^{2} \omega^{2}}<\left(\frac{\Omega}{2}\right)<\omega^{2}+\frac{0.2}{M r^{2}} \sqrt{M^{2} r^{4} \omega^{4}\left(1-\frac{2 r}{R}\right)^{2}-4 n^{2} \omega^{2}} .
$$

Ширина резонансной зоны, в которой положение равновесия $\beta=0$ оказывается неустойчивым и в системе самовозбуждаются колебания, равна

$$
\Delta=\frac{0.4}{M r^{2}} \sqrt{M^{2} r^{4} \omega^{4}\left(1-\frac{2 r}{R}\right)^{2}-4 n^{2} \omega^{2}}
$$

На рис. 4 приведены результаты численного анализа переходного режима колебаний как в непосредственной близости к резонансной зоне, когда расстройка частот $2 \omega-\Omega \neq 0$, так и в самой зоне резонанса. Нутационные колебания носят симметричный характер, происходящие с постоянной фазой. Амплитуда ограничена и стремится к фиксированному значению, т.е. существует устойчивый придельный цикл. Для анализа устойчивостью стационарного режима, т.е. постоянных решений при $\xi_{0}$ образуем уравнения в вариациях, которые приводят к двум условиям устойчивости: $-\frac{2 n}{M r^{2} \omega^{2}}<0, M^{2} r^{4} \omega^{2}\left(1-\frac{2 r}{R}\right)^{2}>4 n^{2}$.Эти условия будут выполнены, если $\omega^{2}=\frac{C_{22}}{M r^{2}}-\frac{r}{R} \Omega^{2}>0$. По соотношениям (4) построены амплитудно-частотные и фазовочастотные характеристики (рис. 3).

Из рис. 3 следует, что существует пороговое значение угловой скорости вращения ротора, при которых наступает резонанс. 

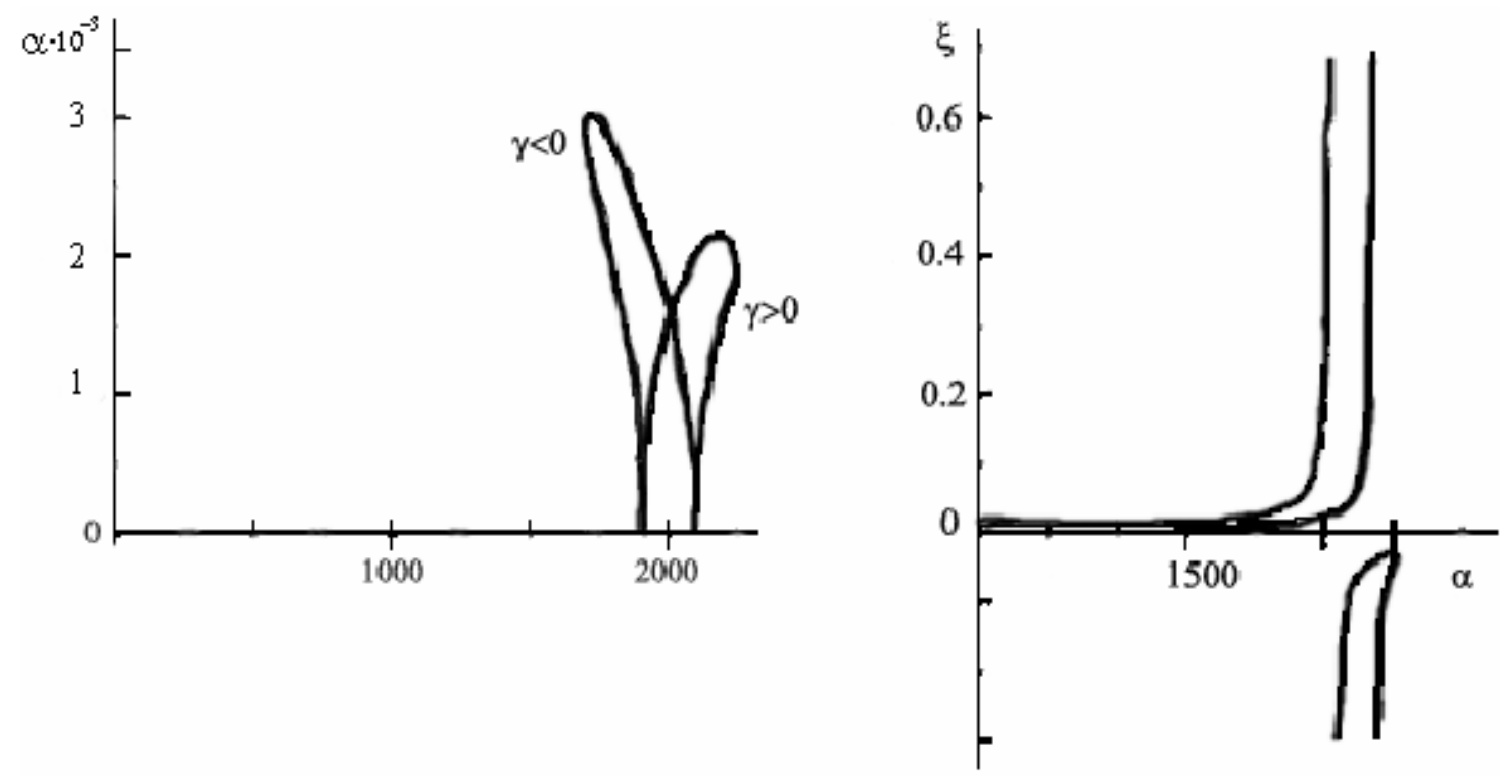

$a$

$\sigma$

Р и с. 3. Амплитудно-частотные (a) и фазово-частотные характеристики (б)

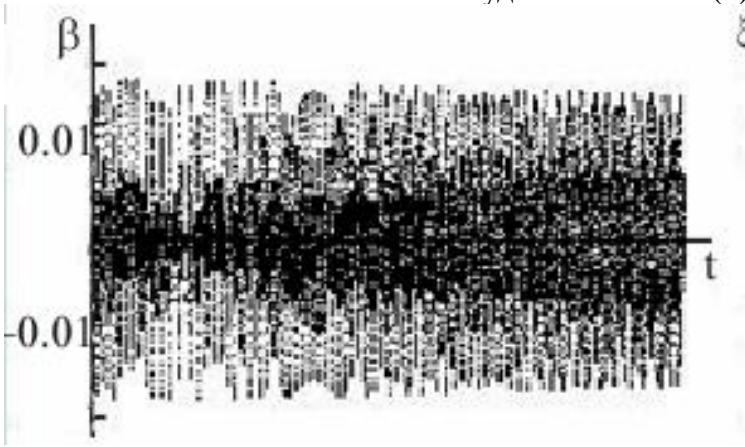

$a$

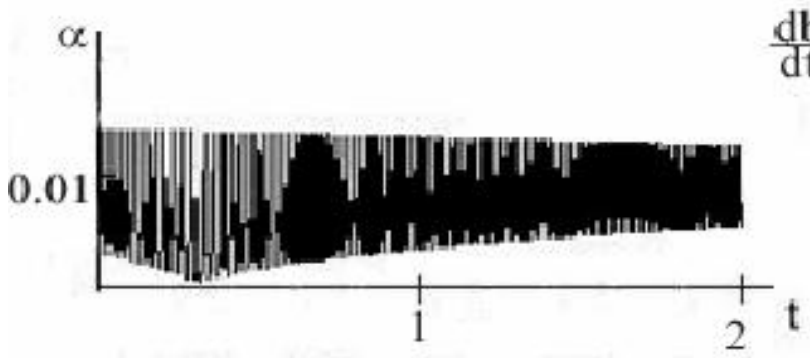

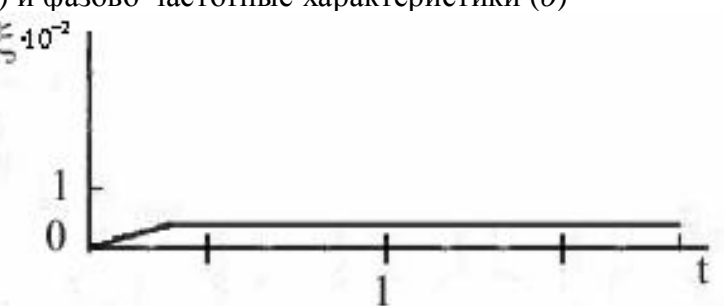

$\sigma$

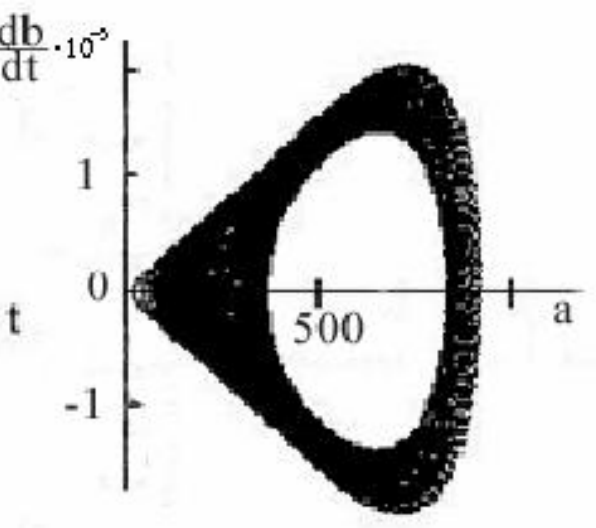
2

Р и с. 4. Численный анализ переходного режима:

$a$ - угол нутации; $\sigma$ - угол $\gamma ; \beta$ - угол прецессии;

2 - фазовая плоскость по амплитуде колебаний ротора

БИБЛИОГРАФИЧЕСКИЙ СПИСОК

1. А.А. Алифов, К.В. Фролов. Взаимодействие нелинейных колебательных систем с источниками энергии. М.: Наука, 1985. 327c.

2. Кельзон А.С., Журавлев Ю.Н., Январев Н.В. Расчет и конструирование роторных машин. Ленинград: Машиностроение, 1977, 287c.

3. Павлов Г.В., Бородин В.С., Вронская Е.С. Динамика вертикального несбалансированного ротора, имеющего точку касания со статором // Труды международного форума по проблемам науки, техники и образования. М., 2001. C 14-16. 